\title{
A review on supervised learning methodologies for detection of exudates in diabetic retinopathy
}

\author{
Ujwala W. Wasekar, R. K. Bathla \\ Department of Computer Science, Desh Bhagat University, Mandi Gobindgarh, Punjab, India
}

\begin{tabular}{l} 
Article Info \\
\hline Article history: \\
Received Mar 18, 2021 \\
Revised Jul 12, 2021 \\
Accepted Jul 18, 2021 \\
\hline
\end{tabular}

\section{Keywords:}

Bright lesions

Deep learning

Fundus image

Image processing

Machine learning

\begin{abstract}
Diabetic retinopathy has become one of the major reasons for blindness in the world. Early and precise diagnosis of the disease may save one's eyesight from irreversible damage. Manual detection of lesions is time consuming and may not be as accurate as desirable. Many automated systems have been developed recently to help ophthalmologists in their endeavors. Exudates are one of the early signs of manifestation of diabetic retinopathy. In this paper, the methodologies detecting exudates in retinal fundus images were reviewed. These methods were categorized into deep learning, machine learning and methods primarily focusing on image processing techniques. The comprehensive view of the performances of the methods was given. Several datasets were described briefly. Most of the researchers preferred combination of multiple publically available databases. Also, the potential areas of research were discussed. It was found that sensitivity which identifies the abnormal images correctly, is the most widely used performance measure. The study will be helpful to the researchers wanting to explore more in this field.
\end{abstract}

This is an open access article under the CC BY-SA license.

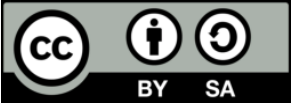

\section{Corresponding Author:}

Ujwala W. Wasekar

Department of Computer Science

Desh Bhagat University

Mandi Gobindgarh, District Fatehgarh Sahib, Punjab - 147301 India

Email: ujwalazade@rediffmail.com

\section{INTRODUCTION}

In the last few years, with the advent of technology, lifestyle of people has changed a lot. Less physical workout and unhealthy eating habits has led to the increased level of glucose in the blood. Since 1990, number of diabetic patients has increased tremendously throughout the globe [1]. Diabetes can give rise to many other disorders such as diabetic retinopathy (DR), diabetic macular edema and glaucoma. DR can directly influence the vision if not treated at an early stage. Manual diagnosis of DR may give inaccurate results. Computer aided screening of DR could be helpful for ophthalmologists in providing quick and precise diagnosis [2]-[4]. Though, therapeutic options such as pharmacotherapy are being made available along with traditional laser therapy [5].

Weakened blood vessels inside the retina rupture resulting into emission of blood and lipids and formation of lesions [6]. The abnormalities that appear on the retina are microaneurysms (MA), haemorrhages (HM) and exudates (EX). Neovascularization (new but abnormal veins) is the apex deformity making precise blood vessel segmentation inevitable [7]. MAs and HMs are called red lesions while EXs are termed as bright lesions. Exudates are again categorized as hard exudates (HE) and cotton wool spots, also known as soft exudates (SE) depending upon their texture and appearance. Some of the early signs of DR are 
microaneurysms and exudates. Figure 1(a) and (b) shows the normal retina and retina having exudates [8]. Normal retina image consists of optic disc (OD), macula and blood vessels.

This paper mainly focuses on the methods available in the literature for the detection and classification of exudates. Exudates appear as bright as optic disc. So, it becomes evident for the researchers to eliminate it before detecting the exudates. Being indicator of the onset of the disease, exudates have to be detected accurately at an early stage to avoid any further complication. There are many techniques proposed in this domain for segmentation, detection and classification of the images as normal and abnormal. In this paper, different methods of evaluation belonging to deep learning, machine learning and image processing, have been reviewed. Each method has its unique way of implementation, but some limitations as well. Various databases and performance metrics have been discussed. All these aspects have been presented in a comprehensive manner.

Rest of the paper is organized as shown in: section 2 presents the databases used in evaluating the algorithm, section 3 briefly describes the performance measures, section 4 provides the different approaches based on deep learning, machine learning and image processing, section 5 gives the outlook of the study under the heading 'discussion', finally, section 6 concludes the work with crisp inference.

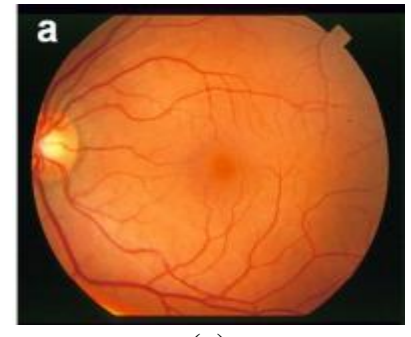

(a)

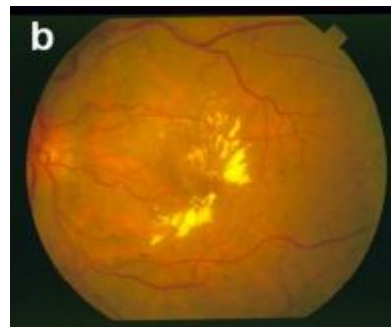

(b)

Figure 1. Show; (a) Normal retina (b) Retina with exudates

\section{DATASETS}

Database is a collection of retinal images that provide a competitive environment for the researchers to conduct fair evaluation of the algorithms. It gives an unambiguous way of assessment of the performance of the methods. There are many publically available datasets with ground truths for the identification and segmentation of various lesions of DR. Some of them are discussed below:

\subsection{Messidor [9]}

It is a publically distributed database containing 1200 fundus images in TIFF format captured using color video $3 \mathrm{CCD}$ camera with $45^{\circ}$ field of view (FOV), 8 bits per color plane. Resolutions of the images were set at $1440 * 960,2240 * 1488$ or $2304 * 1536$ pixels.

\subsection{Diaretdb0 [10]}

It consists of 130 images taken at Kuopio university hospital with $50^{\circ}$ FOV and unknown camera settings. Images are in png format with resolution of $1500 * 1152$ pixels.

\subsection{Diaretdb1 [11]}

It comprises of 89 images in png format. Out of these, 28 and 61 images are for training and testing purpose respectively. Images were taken at $50^{\circ} \mathrm{FOV}$ with size of $1500 * 1152$ pixels.

\subsection{E-ophtha EX [12]}

There are 82 images with $45^{\circ}$ FOV and in JPEG format. Dataset contains images with 4 different sizes ranging from $1440 * 960$ to $2544 * 1696$ pixels.

\subsection{IDRiD [13]}

It has 516 images captured with Kowa VX-10 $\alpha$ digital camera with $50^{\circ}$ FOV. Images are in jpg format with resolution of $4288 * 2848$ pixels. It consists of pixel level annotations for the DR lesions and DR grading at image level. 413 and 103 images make up the training and testing set respectively.

\subsection{Drive [14]}

It contains 40 images with $45^{\circ}$ FOV having size of $768 * 584$ pixels in compressed JPEG format. Images were captured using Canon CR5 nonmydriatic 3CCD camera. 


\subsection{Stare $[15]$}

It consists of 20 images taken by TopCon TRV-50 fundus camera at $35^{\circ} \mathrm{FOV}$ with size of $605^{*} 700$ pixels. Table 1 shows more description about the databases.

Table 1. Description of the databases

\begin{tabular}{lccll}
\hline \multicolumn{1}{c}{ Database } & \multicolumn{2}{c}{ No. of images } & Level of detection & Types of lesions detected \\
& Normal & Abnormal & & \\
\hline Messidor & 546 & 654 & Image & MA, HM, HE, SE \\
Diaretdb0 & 20 & 110 & Image & MA, HM, HE, SE \\
Diaretdb1 & 5 & 84 & Pixel & Exudates \\
e-ophtha EX & 35 & 47 & Pixel & MA, HM, HE, SE \\
IDRiD & 164 (pixel level) & 81 (pixel level) & Pixel, image & Blood vessel \\
Drive & 33 & 7 & Pixel & Blood vessel \\
Stare & 10 & 10 & Pixel & \\
\hline
\end{tabular}

\section{PERFORMANCE METRICS}

Performance measures are the evaluation tools that assist in finding the efficiency of the method or technique. Some widely used measures are sensitivity, specificity and accuracy [2]. Sensitivity is the percentage of correctly identified lesions. Specificity is the percentage of correctly identified non-lesions and accuracy is the average of both. They are given as in:

$$
\begin{array}{ll}
- & \text { Sensitivity }=\frac{T P}{T P+F N} \\
- & \text { Specificity }=\frac{T N}{T N+F P} \\
- & \text { Accuracy }=\frac{T P+T N}{T P+T N+F P+F N}
\end{array}
$$

Where, TP is correctly identified lesions, TN is correctly identified non-lesions, FP is incorrectly identified non-lesions and FN is incorrectly identified lesions.

\section{COMPUTER AIDED SYSTEMS TO CLASSIFY RETINAL IMAGES HAVING EXUDATES}

There are different abnormalities that describe the diabetic retinopathy such as microaneurysms, haemorrhages, exudates and neovascularization. In this paper, we have limited our work to the identification and classification of exudates. Most of the techniques go for widely followed chain of processes viz. preprocessing, segmentation, feature extraction and classification [16]. This section represents the methodologies based on image processing and segmentation, deep learning and machine learning.

\subsection{Image processing and segmentation methodologies}

In image acquisition, some of the noise may get introduced in the image in the form of unwanted pixels, uneven illumination. To get the most out of the image, image should be clear and highlight the objects present in the image. Though, ultra-wide-field images give wider view of retina [17]. Some of the researches have been discussed which primarily focus on the image processing techniques. Noise filtering, contrast enhancement, OD localization [18] are some of the aspects of image processing techniques. Since optic disc resembles exudates in brightness, it is of utmost importance to eliminate it [19]. OD was localized using watershed transformation [20] and exudates were identified with the help of morphological techniques. Method to detect exudates in non-dilated retinal images using mathematical morphology [21] and fuzzy cmeans (FCM) clustering [22] were proposed. Proposed systems may be useful in rural areas where medical facilities are poor. An area-based feature was introduced [23] called as vein removal term (VRT). The method was validated on diaretdb1 database at image level and on e-ophtha EX database at lesion level. Midpoint circle algorithm was used to remove OD. Image was divided and classified into exudate and exudate free. Then the sub image was segmented using saliency method [24]. Considering the time constraint and judicious use of resources, MATLAB and FPGA based solutions have been proposed [25]. The system went through all the requiredpreprocessing techniques. With the use of sensor-based screening systems and cloud software, a telemedicine method [26] was put forward to identify hard exudates in the retinal images. Data was read by d-Eye sensor. Another method used various techniques such as Gamma correction, CLAHE, principal component analysis and convex hull transform [27] for luminosity, contrast enhancement, vessel extraction and OD detection respectively. Morphological operations were used to detect lesions in the fundus image and classification was done based on the count of lesions. A new algorithm [28] called as 'moat operator' based on recursive region growing segmentation algorithms, was developed to recognize HEs, MAs and HMs. Domain knowledge about the lesion was used to correctly identify the exudates [29]. After median filtering and dynamic clustering, domain knowledge was applied to detect true hard exudates. Table 2 depicts the databases used and performances of the above methods. 
Table 2. Performances of methods in detecting exudates using image processing techniques

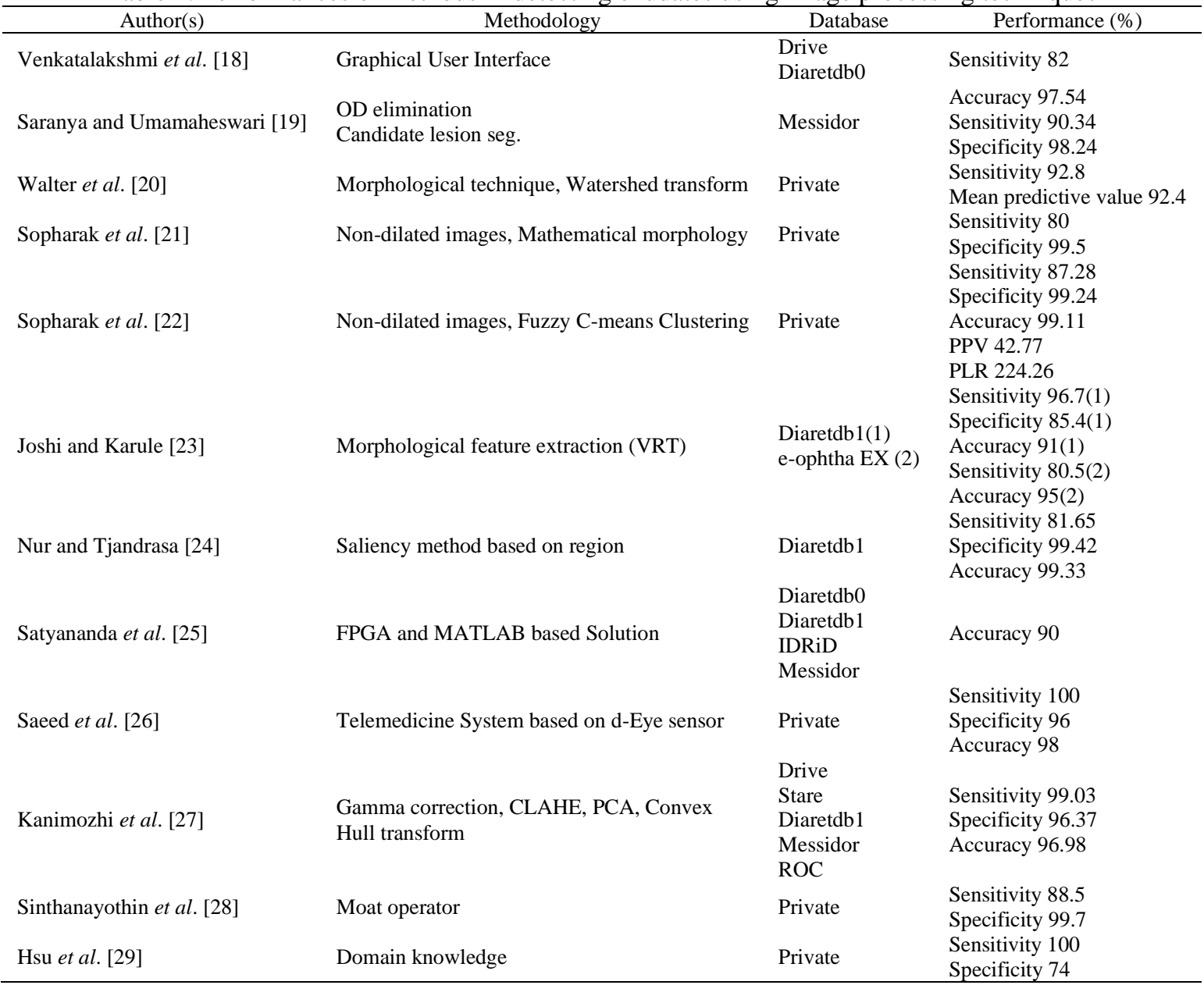

\subsection{Deep learning techniques}

Recently, deep learning (DL) is gaining popularity because of its functionality and applicability. Deep learning is a computer-aided medical diagnosis system [30]. It is a branch of machine learning that consists of nonlinear information processing architectures. It is designed for the purpose of unsupervised feature learning and pattern analysis [31]. Convolutional neural network (CNN) is the most widely used method of DL [32]. The three main layers of CNN include convolution layer, pooling layer and fully connected layer [33].

Much emphasis has been given to the automatic detection of diseases using DL methods recently. An image dictionary was built up with each image of the size $10 * 10$ pixels [34] cut out of the database images. The images were divided into training, testing and validation groups. Using TensorFlow and Keras libraries, a convolutional network in Python was built to detect the exudates. CNNs can also be pretrained to solve the specific problem. Various pretrained CNN models such as Inception-v3, residual network-50 and visual geometry group network-19 were used for feature extraction and classification [35]. Based on transfer learning, the features from all the three models were combined to form a single feature vector (features fusion) and fed to Softmax classifier. Image processing is the first and basic requirement in any pattern detection and classification setup. We have discussed effective image processing techniques in the section 'image processing and segmentation methodologies.' Image processing combined with CNN can do the wonders. Performance of CNN integrated with circular Hough transformation [36] was compared with CNN based performance alone and found out that former gave better results. Another CNN model was trained using patches of size $64 * 64$ pixels extracted from the exudate images [37]. These candidate pixels were taken out using morphological ultimate opening.

A non-linear classification and learning ability make the neural network more efficient. A threelayer perceptron neural network was trained to distinguish between the exudates and non-exudates. After preprocessing, FCM clustering was used for segmentation [38], [39]. Osareh et al. [39], a feature vector was generated with the help of genetic algorithm. Another ANN was used to discriminate between lesions with the help of features such as color, size, shape and texture applying back propagation algorithm [40]. A fuzzy neural network was proposed with self-organizing layer and multilayer perceptron (MLP) [41]. Self-organizing layer 
performed the task of detecting the lesions and grouping them according to their membership values, while MLP network classified them. MLP again proved its superiority in [42] as compared to other three classifiers. Decomposing fundus image into various layers, MLP gave better results on diaretdb1 database [43]. Adaptive neuro-fuzzy inference system (ANFIS) was used to distinguish between normal and abnormal images [44] with texture enhancement using morphological component analysis (MCA). Recently, many new and upgraded approaches emerged as a tool to diagnose lesions such as channel attention method [45], long short-term memory network (LSTM) [46] and capsule network [47]. Working on different databases, these methods gave promising results. More detailed insight of the methodologies is given in Table 3.

Table 3. Exudate detection using deep learning

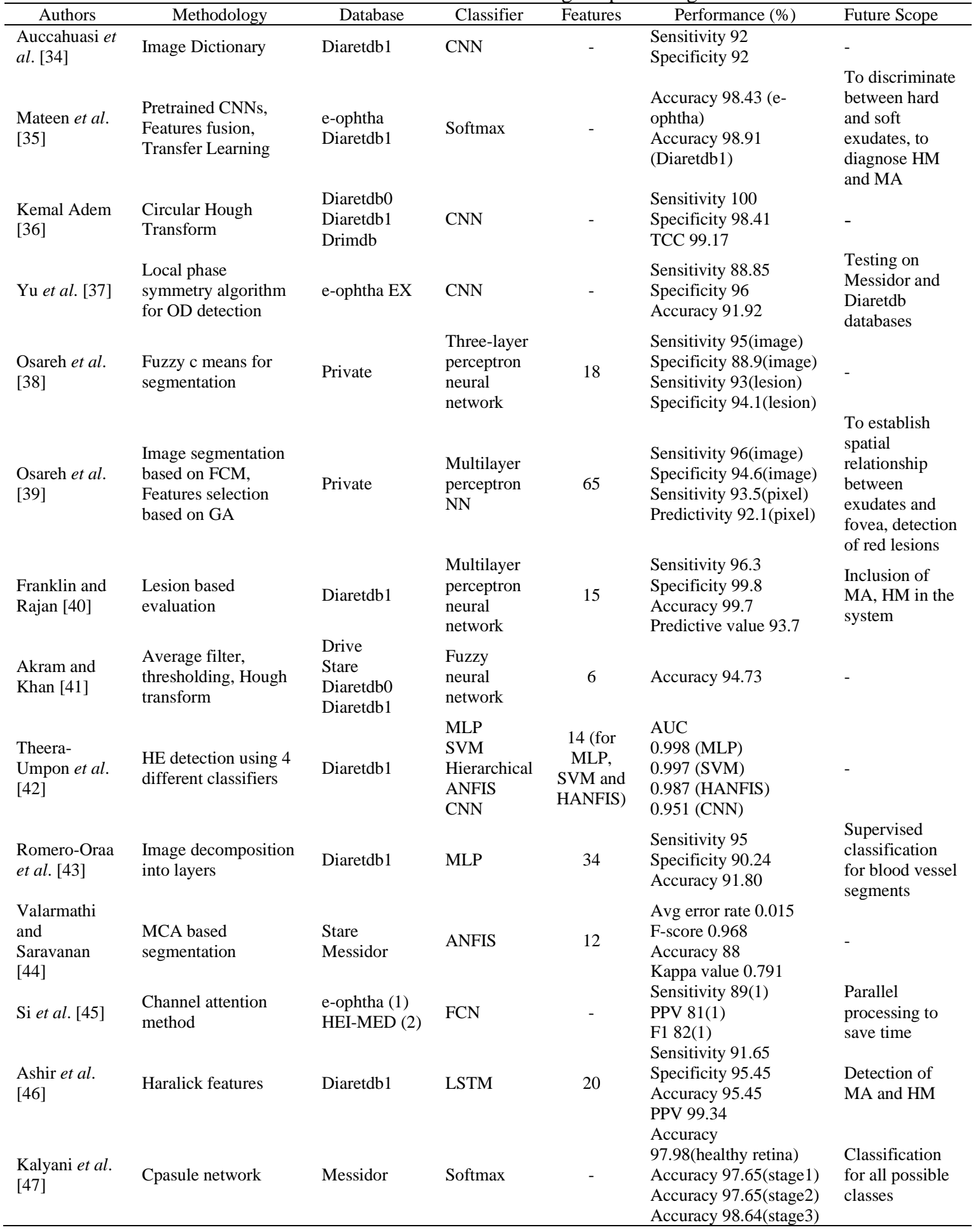




\subsection{Machine learning approach}

Machine learning (ML) is a part of artificial intelligence in which the system learns from data with the intention of analyzing the pattern and take decisions without human intervention. Difference between machine learning and deep learning is illustrated in Table 4 [33].

Table 4. Difference between machine learning and deep learning

\begin{tabular}{lll}
\hline & \multicolumn{1}{c}{ Machine learning } & \multicolumn{1}{c}{ Deep learning } \\
\hline Hand-drawn feature extraction & Required & Not required \\
Training data & Large data not required & Large data required \\
\hline
\end{tabular}

Research in the field of ML includes many state-of-the-art approaches. Support vector machine (SVM) is a ML method specifically used for binary classification. Exudates were distinguished using SVM following some preprocessing techniques. Lesions were detected with the help of morphological opening and closing [48]. SVM based on radial basis function (RBF) detected the hard exudates [49]. It is a common practice to follow the conventional process for detection of lesions using CAD systems i.e. preprocessing, segmentation, feature extraction and classification [50]. Based on morphological operators and texture feature analysis, SVM was trained to identify the images as normal and abnormal [51]. Drusen is an abnormality that appears on retina and resembles to exudates. It is the lesion related to macular degeneration. Its detection is necessary in order to identify exudates correctly. SVM with RBF was trained with features like size, area, shape, colour, brightness and contextual information to grade candidates as exudates, drusen or background [52]. In [53], SVM was tailored for lesion detection with features extracted using local binary patterns (LBP). LBP and granulometric profiles were used [54] to take out features to feed to random forest (RF), SVM and gaussian processes for classification (GPC).

Feature selection using grey wolf optimization was performed and fed to $\mathrm{kNN}$ classifier to discriminate the exudates between proliferative and non-proliferative [55]. A bag of words approach was proposed in which features from image patches were stored to create the dictionary [56]. This feature set was utilized to grade patches between normal, exudate or drusen. A fuzzy logic-based categorization of hard exudates was put forward in which values of hard exudates in RGB color space were used to form the fuzzy set [57], [58]. In another attempt to classify between exudates, gaussian mixture model (GMM) was used. To detect all possible candidate lesions, Gabor filter bank [8] was applied. Retina of younger patients shows higher reflectance because of Nerve Fiber Layer. This issue was tackled by [59] wherein classifier (Fisher's linear discriminant classifier) was trained using texture descriptors. In 2020, Wang, et al. [60], a method is represented that combines deep features with hand crafted ones and classify using RF. Table 5 exhibits the performance, database and other details of the above-mentioned methods.

Table 5. Machine learning methods for exudate detection

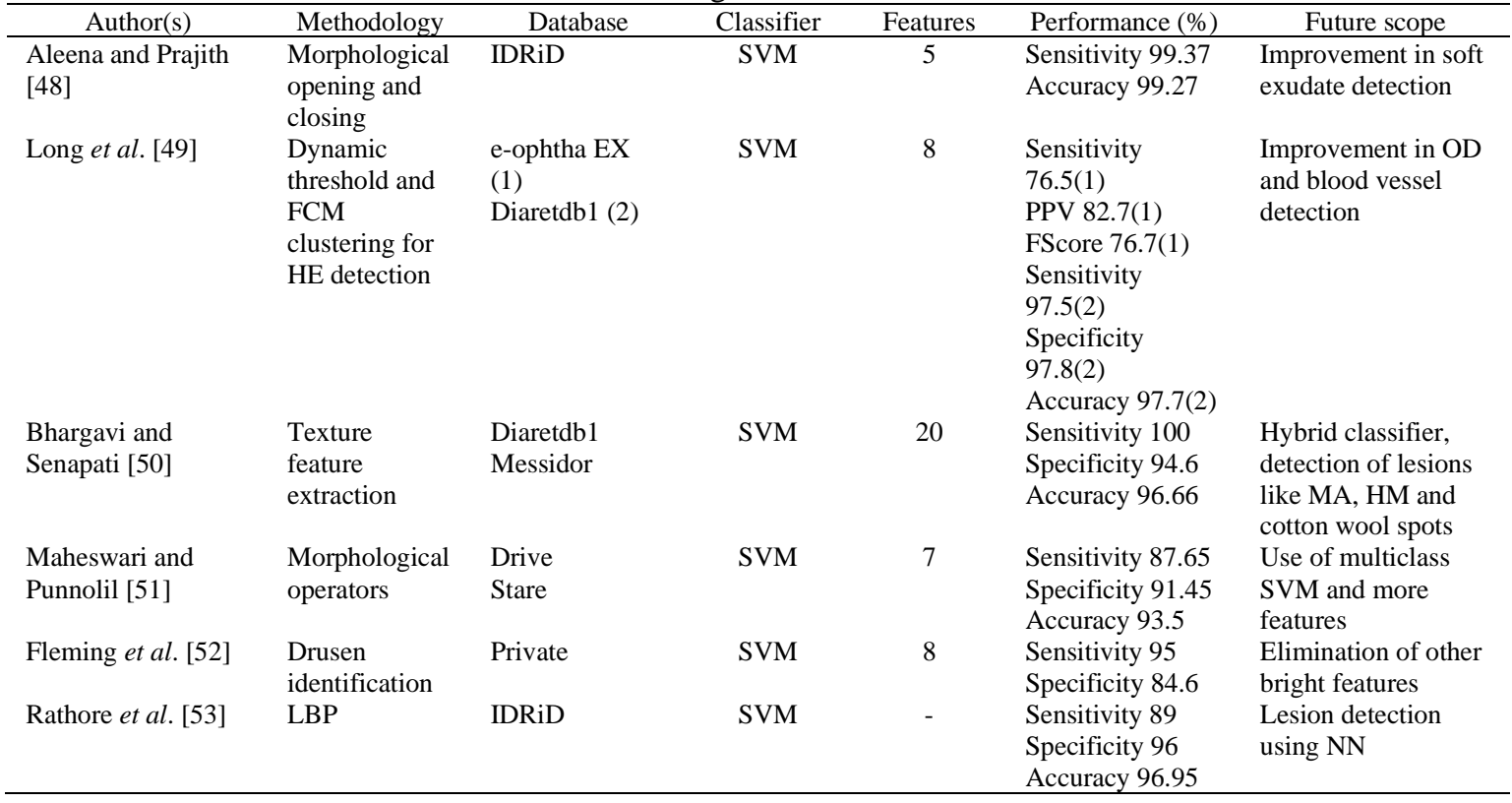


Table 5. Machine learning methods for exudate detection (continue)

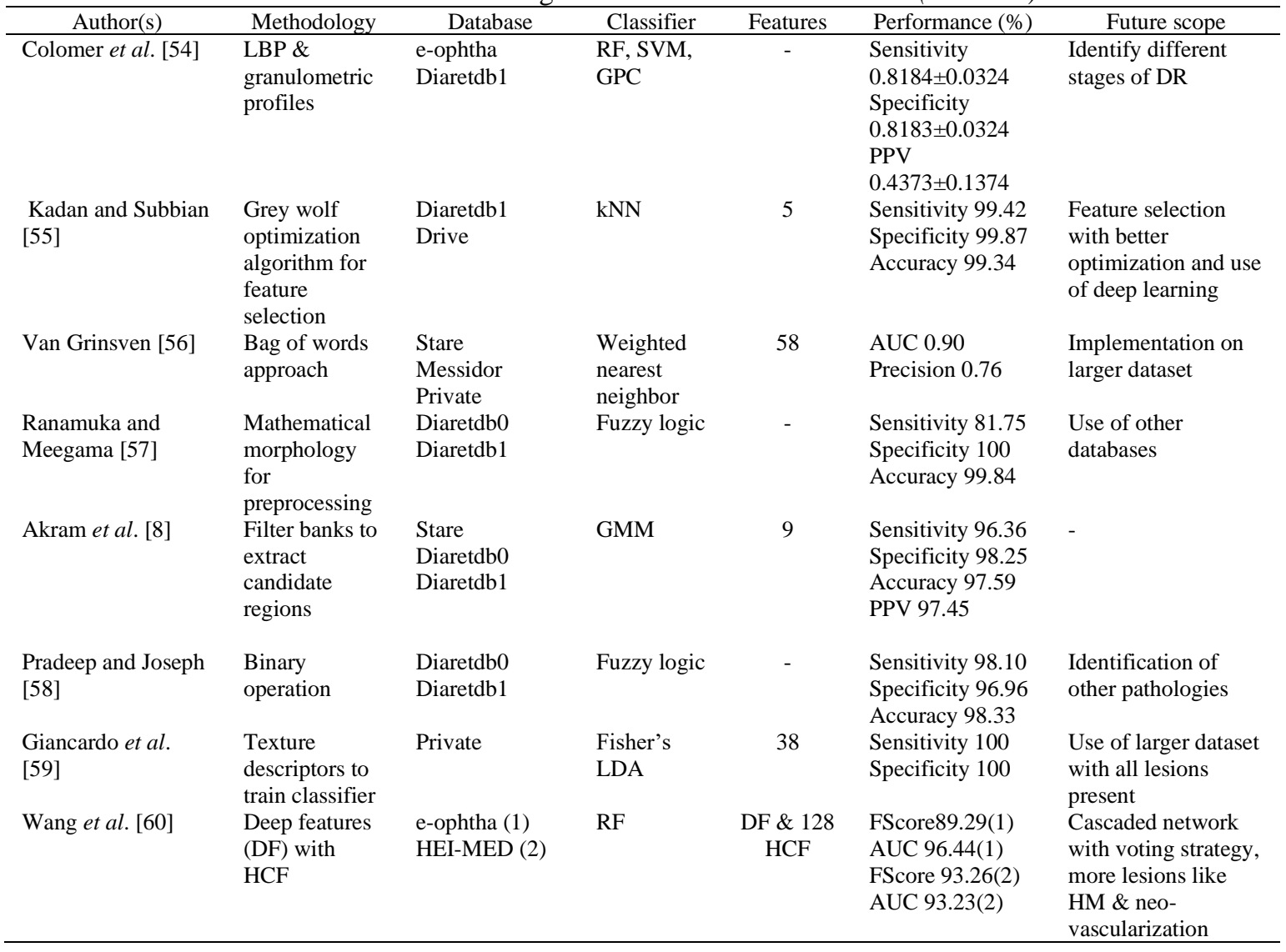

\section{DISCUSSION}

Diabetic retinopathy can claim a permanent vision loss in case of severity. To avoid this, early detection of exudates become of prime concern. Many authors have put forward the appreciable automated systems that could lessen the burden of eye care practitioners and extend the great help to the research community. For successful detection of exudates, every facet in the process should be taken into consideration. Many researches in the domain of medical diagnosis of the bright lesions in DR images have been illustrated. Methodologies were categorized pertaining to deep learning, machine learning and those mainly focusing on image processing techniques. Methods used for preprocessing play a vital role in refining the image in turn extracting more beneficial information for pattern recognition. Since, OD is as bright as exudate, its elimination ensures smooth identification of exudate. Various approaches are available for OD detection such as local phase symmetry [37] and Hough transform [36], [41]. Fuzzy c means for segmentation [22], [38], [39], [49] is widely used. Morphological operators (in association with structuring elements) make its place in almost every methodology [20], [48], [51], [57] because of its ability to segment the objects in the image depending on their shapes.

Deep learning is a branch of machine learning and requires larger data to train the system [33]. Building a neural network may be time consuming and effort demanding. Instead, pretrained systems can be utilized [35]. Parallel processing systems may also boost the performance by saving time [45]. DL combined with image processing may prove efficient set-up [36]. Relevant features of an entity can accurately identify it. Exudates can be greatly recognized by its shape, size, color, texture, intensity and edge strength [8], [38], [55], [44]. Detection of all lesions [46], [58] and multiple DR stages [47], [54] is the matter of concern.

Multiple databases are publically available for the researchers that provide absolute uniform platform to evaluate the methods proposed by them. Several authors opt for private data i.e. data taken from some health care institutions and evaluation done on the basis of the hand-drawn ground truth given by the experts [21], [39], [59]. In this study one fourth of the methodologies used private datasets. Figure 2 depicts the distribution of databases according to their domain and multiplicity. It is clear that many authors prefer the combination of multiple public databases to enhance the performance. Also, public databases give more competitive environment for evaluation. Performance measures offer different angles of assessment of the system and provide incentive to perform better. Figure 3 shows that sensitivity, specificity and accuracy are 
extensively used measures for system evaluation. In medical domain, sensitivity is the most go for measure. Though, investigation in this realm has reached a far way. Still, there is a need to focus on some areas like discriminating between hard and soft exudates [35], better feature selection [55] and improvement in OD and blood vessel detection [49].

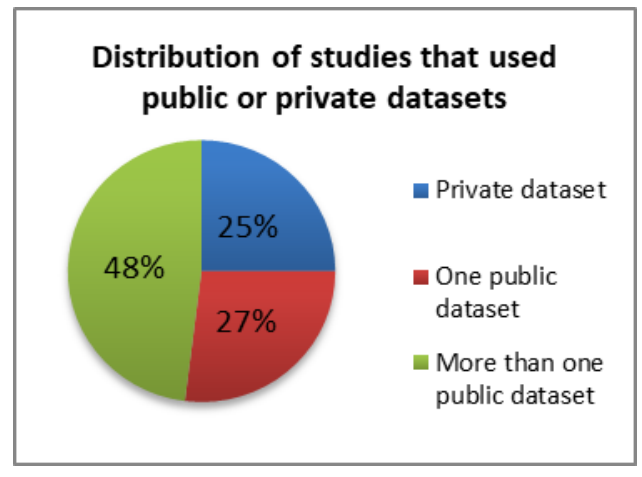

Figure 2. Percentage distribution of databases

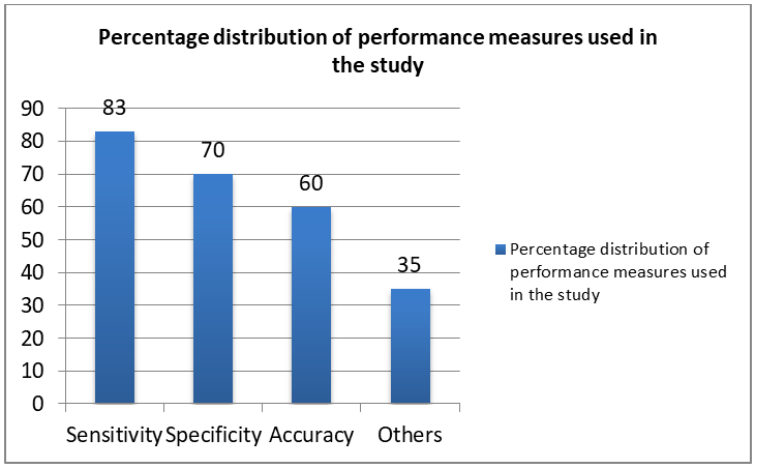

Figure 3. Usage of performance measures in used in the study the study

\section{CONCLUSION}

Diabetic Retinopathy has become the major concern for visual impairment worldwide. Recently computer aided diagnosis (CAD) systems along with image processing techniques have emerged out to be beneficial in terms of cost, time and accuracy. Many state-of-the-art approaches have been proposed in this regard. According to British Diabetic Association, minimum 80\% sensitivity and 95\% specificity is desirable. Various methodologies pertaining to classification modules such as deep learning and machine learning have been briefly discussed. It is observed that SVM is the popular approach in machine learning. Methods suggested by the researchers are efficient but have potential areas for experimentation. Deep learning with image processing could be a considerable area to explore. Since deep learning requires a large amount of data and significant time to build, step can be put forward to address these issues. Several publically available databases were briefly described. Selection of multiple datasets is a common choice for many researchers. It could be inferred that sensitivity is the widely used measure. Comparing the performances of the algorithms instigate to perform better to give maximum throughput. However, the most efficient and capable automated system is still on the way.

\section{REFERENCES}

[1] X. Lin, et al., "Global, regional, and national burden and trend of diabetes in 195 countries and territories: an analysis from 1990 to 2025," Scientific Reports, vol. 10, no. 1, Sept. 2020, doi: 10.1038/s41598-020-71908-9.

[2] R. S. Biyani and B. M. Patre, "Algorithms for red lesion detection in Diabetic Retinopathy: A review," Biomedicine \& Pharmacotherapy, vol. 107, pp. 681-688, Nov. 2018, doi: 10.1016/j.biopha.2018.07.175.

[3] S. Joshi and P. T.Karule, "A review on exudates detection methods for diabetic retinopathy," Biomedicine \& Pharmacotherapy, vol. 97, pp. 1454-1460, Jan. 2018, doi: 10.1016/j.biopha.2017.11.009.

[4] H. Asha, et al., "Detection and grading of diabetic retinopathy in retinal images using deep intelligent systems: a comprehensive review," Computers, Materials \& Continua, vol. 66, no. 3, pp. 2771-2786, 2021, doi: $10.32604 / \mathrm{cmc} .2021 .012907$.

[5] S. E. Mansour, D. J. Browning, K. Wong, H. W. Flynn Jr, and A. R. Bhavsar, "The Evolving Treatment of Diabetic Retinopathy," Clinical Ophthalmology, vol. 14, pp. 653-678, 2020, doi: 10.2147/OPTH.S236637.

[6] A. Issac, M. K. Dutta, and C. M. Travieso, "Automatic computer vision-based detection and quantitative analysis of indicative parameters for grading of diabetic retinopathy," Neural Computing and Applications, vol. 32, pp. 15687-15697, 2020, doi: 10.1007/s00521-018-3443-z.

[7] M. U. Akram, I. Jamal, and A. Tariq, "Blood Vessel Enhancement and Segmentation for Screening of Diabetic Retinopathy," TELKOMNIKA Telecommunication, Computing, Electronics and Control, vol. 10, no. 2, pp. 327-334, Jun 2012, doi: 10.11591/telkomnika.v10i2.686.

[8] M. U. Akram, A. Tariq, M. A. Anjum, and M. Y. Javed, "Automated detection of exudates in colored retinal images for diagnosis of diabetic retinopathy," Applied Optics, vol. 51, no. 20, pp. 4858-4866, Jul. 2012, doi: 10.1364/AO.51.004858.

[9] E. Decencière, et al., "Feedback on a publicly distributed image database: the messidor database," Image Analysis \& Stereology, vol. 33, no. 3, pp. 231-234, 2014, doi: 10.5566/ias.1155.

[10] T. Kauppi, et al., "DIARETDB0: Evaluation Database and Methodology for Diabetic Retinopathy Algorithms," Technical report, vol. 73, pp. 1-17, 2006. 
[11] T. Kauppi, et al., "Diaretdb1 diabetic retinopathy database and evaluation protocol," Proceedings Medical Image Understanding and Analysis (MIUA), vol. 2007, 2007, doi: 10.5244/C.21.15.

[12] X. Zhang, et al., "Exudate detection in color retinal images for mass screening of diabetic retinopathy," Medical Image Analysis, vol. 18, no. 7, pp. 1026-1043, Oct. 2014, doi: 10.1016/j.media.2014.05.004.

[13] P. Porwal, et al., "Indian Diabetic Retinopathy Image Dataset (IDRiD): A Database for Diabetic Retinopathy Screening Research,” Data, vol 3, no. 3, 2018, doi: 10.3390/data3030025.

[14] J. Staal, M. D. Abramoff, M. Niemeijer, M. A. Viergever, and B.van Ginneken, "Ridge-Based Vessel Segmentation in Color Images of the Retina," IEEE Transactions on Medical Imaging, vol.23, no. 4, pp. 501-509, Apr. 2004, doi: 10.1109/TMI.2004.825627.

[15] A. Hoover, V. Kouznetsova, and M. Goldbaum, "Locating blood vessels in retinal images by piecewise threshold probing of a matched filter response," IEEE Transactions on Medical Imaging, vol. 19, no. 3, pp. 203-210, 2000, doi: $10.1109 / 42845178$

[16] G. T. Zago, R. V. Andreão, B. Dorizzi, and E. O. Teatini Salles, "Diabetic retinopathy detection using red lesion localization and convolutional neural networks," Computers in Biology and Medicine, vol. 116, Jan. 2020, doi: 10.1016/j.compbiomed.2019.103537.

[17] K. Oh, H. M. Kang, D. Leem, H. Lee, K. Y. Seo, and S. Yoon, "Early detection of diabetic retinopathy based on deep learning and ultra-wide-field fundus images," Scientific Reports, vol. 11, no. 1, 1897, Jan. 2021, doi: $10.1038 / \mathrm{s} 41598-021-81539-3$.

[18] B. Venkatalakshmi, V. Saravanan, and G. J. Niveditha, "Graphical user interface for enhanced retinal image analysis for diagnosing diabetic retinopathy," 2011 IEEE 3rd International Conference on Communication Software and Networks, 2011, pp. 610-613, doi: 10.1109/ICCSN.2011.6014967.

[19] P. Saranya and K. M. Umamaheswari, "Detecting Exudates in Color Fundus Images for Diabetic Retinopathy Detection Using Deep Learning," Annals of the Romanian Society for Cell Biology, vol. 25, no. 5, pp. 5368-5375, 2021.

[20] T. Walter, J. Klein, P. Massin, and A. Erginay, "A contribution of image processing to the diagnosis of diabetic retinopathy-detection of exudates in color fundus images of the human retina," IEEE Transactions on Medical Imaging, vol. 21, no. 10, pp. 1236-1243, Oct. 2002, doi: 10.1109/TMI.2002.806290.

[21] A. Sopharak, B. Uyyanonvara, S. Barman, and T. H. Williamson, "Automatic detection of diabetic retinopathy exudates from non-dilated retinal images using mathematical morphology methods," Computerized Medical Imaging and Graphics, vol. 32, no. 8, pp. 720-727, Dec. 2008, doi: 10.1016/j.compmedimag.2008.08.009.

[22] A. Sopharak, B. Uyyanonvara, and S. Barman, "Automatic Exudate Detection from Non-dilated Diabetic Retinopathy Retinal Images Using Fuzzy C-means Clustering,” Sensors, vol. 9, no. 3, pp. 2148-2161, Mar. 2009, doi: 10.3390/s90302148.

[23] S. Joshi and P. T. Karule, "Detection of Hard Exudates Based on Morphological Feature Extraction," Biomed Pharmacol J, vol. 11, no. 1, pp. 215-225, 2018, doi: 10.13005/bpj/1366.

[24] N. Nur and H. Tjandrasa, "Exudate Segmentation in Retinal Images of Diabetic Retinopathy Using Saliency Method Based on Region,” J. of Phys.: Conf. Series, vol. 1108, 2018, doi: 10.1088/1742-6596/1108/1/012110.

[25] V. Satyananda, K. V. Narayanaswamy, and Karibasappa, "FPGA and MATLAB Based Solution for Retinal Exudate Detection," International Journal of Recent Technology and Engineering, vol. 8, no. 6, pp. 727-734, 2020, doi: 10.35940/ijrte.

[26] E. Saeed, M. Szymkowski, K. Saeed, and Z. Mariak, "An Approach to Automatic Hard Exudate Detection in Retina Color Images by a Telemedicine System Based on the d-Eye Sensor and Image Processing Algorithms," Sensors, vol. 19, no. 3, 2019, doi: 10.3390/s19030695.

[27] J. Kanimozhi, P. Vasuki, and S. M. M. Roomi, "Fundus image lesion detection algorithm for diabetic retinopathy screening," Journal of Ambient Intelligence and Humanized Computing, vol. 12, pp. 7407-7416, 2020, doi: https://doi.org/10.1007/s12652-020-02417-w.

[28] C. Sinthanayothin, et al., "Automated detection of diabetic retinopathy on digital fundus images," Diabetic Medicine, vol.19, no. 2, pp. 105-112, Feb. 2002, doi: 10.1046/j.1464-5491.2002.00613.x.

[29] W. Hsu, P. M. D. S. Pallawala, M. Li Lee, and K. Au Eong, "The role of domain knowledge in the detection of retinal hard exudates," Proceedings of the 2001 IEEE Computer Society Conference on Computer Vision and Pattern Recognition. CVPR 2001, 2001, pp. II-II, doi: 10.1109/CVPR.2001.990967.

[30] D. Lin, A. V. Vasilakos, Y. Tang, and Y. Yao, "Neural networks for computer-aided diagnosis in medicine: A review," Neurocomputing, vol. 216, pp. 700-708, Dec. 2016, doi: 10.1016/j.neucom.2016.08.039.

[31] L. Deng, "A tutorial survey of architectures, algorithms, and applications for deep learning," APSIPA Transactions on Signal and Information Processing, vol. 3, 2014, doi: 10.1017/atsip.2013.9.

[32] M. Bakator and D. Radosav, "Deep Learning and Medical Diagnosis: A Review of Literature," Multimodal Technologies and Interaction, vol. 2, no. 3, 2018, doi: 10.3390/mti2030047.

[33] W. L. Alyoubi, W. M. Shalash, and M. F. Abulkhair, "Diabetic retinopathy detection through deep learning techniques: A review," Informatics in Medicine Unlocked, vol. 20, 2020, doi: 10.1016/j.imu.2020.100377.

[34] W. Auccahuasi, et al., "Recignition of hard exudates using Deep Learning," in International Conference on Computational Intelligence and Data Science, vol. 167, 2019, pp. 2343-2353, doi: 10.1016/j.procs.2020.03.287.

[35] M. Mateen, J. Wen, N. Nasrullah, S. Sun, and S. Hayat, "Exudate Detection for Diabetic Retinopathy Using Pretrained Convolutional Neural Networks," Complexity, vol. 2020, pp. 1-11, 2020, doi: 10.1155/2020/5801870.

[36] K. Adem, "Exudate detection for diabetic retinopathy with circular Hough transformation and convolutional neural networks," Expert Systems with Applications, vol. 114, pp. 289-295, Dec. 2018, doi: 10.1016/j.eswa.2018.07.053. 
[37] S. Yu, D. Xiao, and Y. Kanagasingam, "Exudate detection for diabetic retinopathy with convolutional neural networks," in 2017 39th Annual International Conference of the IEEE Engineering in Medicine and Biology Society (EMBC), 2017, pp. 1744-1747, doi: 10.1109/embc.2017.8037180.

[38] A. Osareh, M. Mirmehdi, B. Thomas, and R. Markham, "Automated identification of diabetic retinal exudates in digital colour images," British Journal of Ophthalmology, vol. 87, no. 10, pp. 1220-1223, 2003, doi: $10.1136 /$ bjo.87.10.1220.

[39] A. Osareh, B. Shadgar, and R. Markham, "A Computational-Intelligence-Based Approach for Detection of Exudates in Diabetic Retinopathy Images," IEEE Transactions on Information Technology in Biomedicine, vol. 13, no. 4, pp. 535-545, Jul. 2009, doi: 10.1109/TITB.2008.2007493.

[40] S. W. Franklin and S. E. Rajan, "Diagnosis of diabetic retinopathy by employing image processing technique to detect exudates in retinal images," IET Image Processing, vol. 8, no. 10, pp. 601-609, Oct. 2014, doi: 10.1049/ietipr.2013.0565.

[41] U. M. Akram and S. A. Khan, "Automated Detection of Dark and Bright Lesions in Retinal Images for Early Detection of Diabetic Retinopathy," Journal of Medical Systems, vol. 36, no. 5, pp. 3151-3162, Oct. 2012, doi: 10.1007/s10916-011-9802-2.

[42] N. Theera-Umpon, I. Poonkasem, S. Auephanwiriyakul, and D. Patikulsila, "Hard exudate detection in retinal fundus images using supervised learning," Neural Computing and Applications, vol. 32, pp. 13079-13096, 2020 , doi: 10.1007/s00521-019-04402-7.

[43] R. Romero-Oraá, M. García, J. Oraá-Pérez, M. I. López-Gálvez and R. Hornero, "Effective Fundus Image Decomposition for the Detection of Red Lesions and Hard Exudates to Aid in the Diagnosis of Diabetic Retinopathy," Sensors, vol. 20, no. 22, 2020, doi: 10.3390/s20226549.

[44] R. Valarmathi and S. Saravanan, "Exudate characterization to diagnose diabetic retinopathy using generalized method," Journal of Ambient Intelligence and Humanized Computing, vol. 12, pp. 3633-3645, Mar. 2021, doi: 10.1007/s12652-019-01617-3.

[45] Z. Si, D. Fu, Y. Liu, and Z. Huang, "Hard exudate segmentation in retinal image with attention mechanism," IET Image Process, vol.15, no. 3, pp. 587- 597, Feb. 2021, doi: 10.1049/ipr2.12007.

[46] A. M. Ashir, S. Ibrahim, M. Abdulghani, A. A. Ibrahim, and M. S. Anwar, "Diabetic Retinopathy Detection Using Local Extrema Quantized Haralick Features with Long Short-Term Memory Network," International Journal of Biomedical Imaging, vol. 2021, 2021, doi: 10.1155/2021/6618666.

[47] G. Kalyani, B. Janakiramaiah, A. Karuna, and L. V. Narasimha Prasad, "Diabetic retinopathy detection and classification using capsule networks," Complex \& Intelligent Systems, 2021, doi: 10.1007/s40747-021-00318-9.

[48] S. L. Aleena and C. A. Prajith, "Retinal Lesions Detection for Screening of Diabetic Retinopathy," in 2020 11th International Conference on Computing, Communication and Networking Technologies (ICCCNT), Kharagpur, India, 2020, pp. 1-6, doi: 10.1109/ICCCNT49239.2020.9225617.

[49] S. Long, X. Huang, Z. Chen, S. Pardhan, and D. Zheng, "Automatic Detection of Hard Exudates in Color Retinal Images Using Dynamic Threshold and SVM Classification: Algorithm Development and Evaluation," BioMed Research International, vol. 2019, 2019, doi: 10.1155/2019/3926930.

[50] R. Bhargavi and R. K. Senapati, "Bright lesion detection in color fundus images based on texture features," Bulletin of electrical engineering and informatics, vol. 5, no. 1, pp 92-100, March 2016, doi: 10.11591/eei.v5i1.553.

[51] M. S. Maheswari and A. Punnolil, "A Novel Approach for Retinal Lesion Detection Diabetic Retinopathy Images," in 2014 International Conference on Innovations in Engineering and Technology, India, 2014, vol. 3, no. 3, pp. $1109-1114$

[52] A. D. Fleming, et al., "Automated detection of exudates for diabetic retinopathy screening," Physics in Medicine and Biology, vol. 52, no. 24, pp. 7385-7396, Dec. 2007, doi: 10.1088/0031-9155/52/24/012.

[53] S. Rathore, A. Aswal, and P. Saranya, "Bright Lesion Detection in Retinal Fundus Images for Diabetic Retinopathy Detection Using Machine Learning Approach," Annals of the Romanian Society for Cell Biology, vol. 25, no. 5, pp. $4360-4367,2021$.

[54] A. Colomer, J. Igual, and V. Naranjo, "Detection of Early Signs of Diabetic Retinopathy Based on Textural and Morphological Information in Fundus Images," Sensors, vol. 20, no. 4, Feb. 2020, doi: 10.3390/s20041005.

[55] A. B. Kadan and P. S. Subbian, "Detection of Hard Exudates Using Evolutionary Feature Selection in Retinal Fundus Images," Journal of Medical Systems, vol. 43, 2019, doi: 10.1007/s10916-019-1349-7.

[56] M. J. J. P. van Grinsven, A. Chakravarty, J. Sivaswamy, T. Theelen, B. van Ginneken, and C. I. Sánchez, "A Bag of Words approach for discriminating between retinal images containing exudates or drusen," 2013 IEEE 10th International Symposium on Biomedical Imaging, 2013, pp. 1444-1447, doi: 10.1109/ISBI.2013.6556806.

[57] N. G. Ranamuka and R. G. N. Meegama, "Detection of hard exudates from diabetic retinopathy images using fuzzy logic," IET Image Processing, vol.7, no. 2, pp. 121-130, Mar. 2013, doi: 10.1049/iet-ipr.2012.0134.

[58] A. Pradeep and F. Joseph, "Binary operation based hard exudate detection and fuzzy based classification in diabetic retinal fundus images for real time diagnosis applications," International Journal of Electrical and Computer Engineering (IJECE), vol. 10, no. 3, pp. 2305-2312, 2020, doi: 10.11591/ijece.v10i3.pp2305-2312.

[59] L.Giancardo, et al., "Bright Retinal Lesions Detection using Color Fundus Images Containing Reflective Features," in World Congress on Medical Physics and Biomedical Engineering, Munich, Germany, 2009, pp. 292-295, doi: 10.1007/978-3-642-03891-4_78.

[60] H. Wang, et al., "Hard exudate detection based on deep model learned information and multi-feature joint representation for diabetic retinopathy screening,"Computer Methods and Programs in Biomedicine, vol. 191, Jul. 2020, doi: 10.1016/j.cmpb.2020.105398. 